\title{
Neglected Posterior Knee Dislocation: An Unusual Case Report
}

\author{
Francesco Leonardi ${ }^{1} \quad$ Alessandra Zorzan ${ }^{2} \quad$ Augusto Palermo $^{3} \quad$ Luigi Molfetta ${ }^{4}$ \\ 1 Orthopaedic Unit, Policlinic Institute, Alessandria, Italy \\ ${ }^{2}$ Orthopaedic Unit, Hospital of Savigliano, Cuneo, Italy \\ ${ }^{3}$ Italian Auxologic Institute IRCSS, Capitanio Clinic, Milan, Italy \\ ${ }^{4}$ Department of Internal Medicine, School of Medical and

\begin{abstract}
Address for correspondence Luigi Molfetta, Department of Internal Medicine, School of Medical and Pharmaceutical Sciences, University of Genoa, Via Laggiaro 35, 16035 Rapallo, Genoa, Italy
\end{abstract} \\ (e-mail: Imolfetta@libero.it).
}

Pharmaceutical Sciences, University of Genoa, Genoa, Italy

Joints 2017;5:253-255.

\begin{abstract}
Keywords

- knee

- dislocation

- chronic

- neglected

- neurovascular

- treatment

Chronic dislocation of the knee is a rare condition due to a diagnostic mistake during emergency assessment. Usually, dislocation obliges the surgeon to carry out a broad assessment, with vascular and neurological examination, and a thorough assessment of joint injuries. The immediate and stable closed reduction can resolve neurovascular compression. The most serious late sequela is joint instability with impairment of ambulation and patient's autonomy. Treatment of neglected knee dislocation is surgical, that is, arthrodesis or arthroplasty, the latter representing the treatment of choice. The case described herein appears unique because of the clinical and radiographic features. Interestingly, over the years, the neurovascular bundle has inadvertently adapted to the posterior femur displacement without secondary damage.
\end{abstract}

\section{Introduction}

Traumatic knee dislocation is a severe although rare injury, evaluated by $0.02 \%$ to the skeletal trauma, albeit the true incidence is unknown as often reduction of the dislocation is performed at the scene of the trauma. It is often caused by a severe trauma, although rare cases have been described due to nonsevere traumas, such as hyperextension.

Knee dislocation is an orthopaedic emergency because of the possible capsular ligamentous, neurovascular injuries, compartment syndrome, or open lesions. The incidence of neurovascular damage is estimated by $20 \%$ of cases. Femoral nerve palsy and popliteal artery early disruption are the most frequent early complications with a variable incidence of 10 and $25 \%$, respectively. The most common late complications are instability and limping. ${ }^{1}$

Knee dislocation is classified, according to tibial displacement, as posterior, medial, and lateral, with a rotatory and anterior component as the most frequent complication. ${ }^{2}$ It requires an emergency treatment to achieve immediate and stable reduction and to treat any associated injury, especially neurovascular ones. Surgical treatment is necessary in irreducible dislocations, in open injuries, and when the neurovascular structures are compromised.
Rare cases of inveterate and chronic dislocation of the knee have been described; these are difficult to classify because of the variability of lesion patterns and/or of the associated injuries. ${ }^{3}$ The case that we describe herein shows many peculiarities concerning the etiology, the pathologic features, and the absence of neurovascular injuries caused by the injury itself, but with progressive adaptation of the neurovascular bundle to the pathological condition without additional late damage.

\section{Case Report}

This is the case of a 69-year-old woman, weighing $130 \mathrm{~kg}$, wearing a knee brace on her right lower limb, due to deformation, instability, and load-dependent pain. She had an ambulatory range limited to a few steps and therefore made use of a wheelchair.

She accidentally fell at home and hurt her right knee, with a reported trauma in hyperextension. X-rays revealed a subluxation with articular diastasis and a concomitant detachment of the apex of the fibular head. This was the only lesion identified and treated by the orthopaedic surgeon at the time of the first visit.

Her leg was placed into a knee plaster cast with no weight bearing for 30 days. Once the cast was removed, X-rays published online October 4, 2017
DOI https://doi.org/ 10.1055/s-0037-1607219. ISSN 2282-4324.
Copyright @ 2017 Georg Thieme Verlag KG Stuttgart · New York

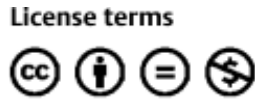




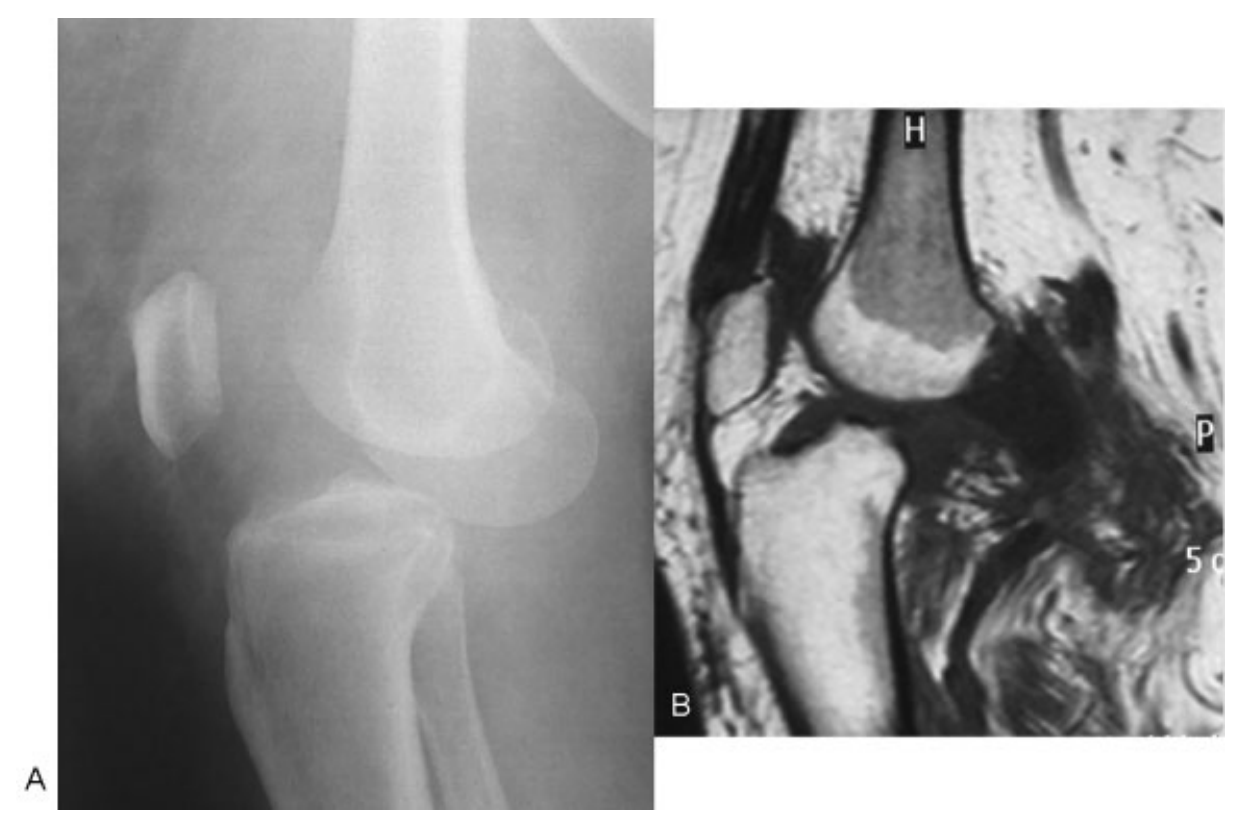

Fig. 1 Imaging after the plaster has been removed. (A) Persistent joint diastasis and onset of a posterior subluxation of the femur can be seen on $\mathrm{X}$-rays. (B) Magnetic resonance image shows integrity of the extensor mechanism.

revealed the persistence of an articular diastasis and severe anterior subluxation of the tibia. She was prescribed an articulated brace, rehabilitation, and progressive recover of weight bearing. Doppler ultrasound of the lower limbs was negative, and magnetic resonance (MR) revealed no conditions worthy of urgent treatment. No surgical treatment was suggested and the patient was left with her articular disease, to which she adapted without any further complications (-Fig. 1). She came to our attention 3 years after the trauma, with an X-ray that showed a total and inveterate dislocation of her right knee. MR exam documented severe alterations of the soft tissues, although there was no apparent discontinuity of extensor apparatus. Computed tomography angiography with contrast medium was highly significant and showed the unexpected adaptation of the neurovascular structures to the total dislocation (-Fig. 2).

At the clinical exam, the knee showed a severe multidirectional instability; the dislocation was irreducible, but the patient showed 90 degrees of knee flexion and full active extension, which confirmed the integrity of the extensor apparatus. There was tolerable pain, only during active extension.

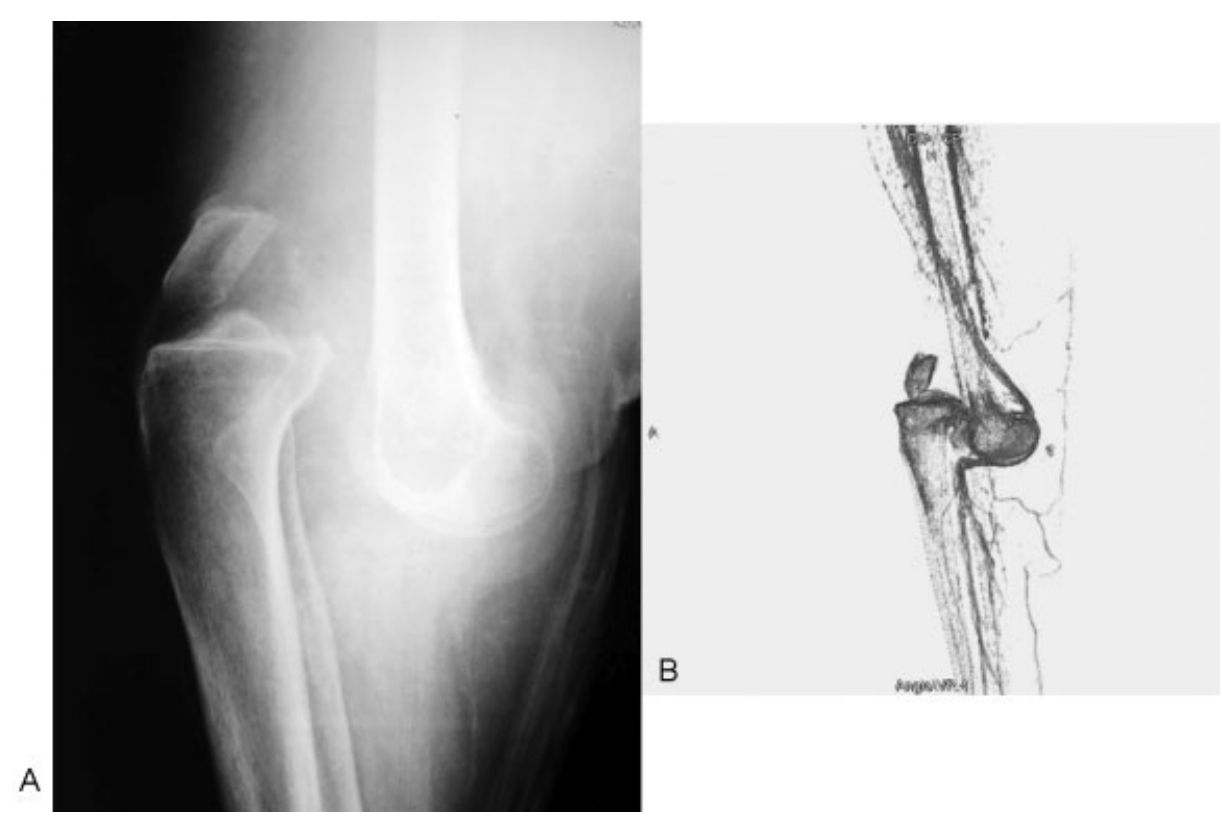

Fig. 2 Imaging at 2-year follow-up. (A) X-ray shows complete anterior dislocation of the tibia. (B) Computed tomography angiography documents the dislocation and the kinking of the popliteal vascular fascia in absence of significant distal stenosis. 
The arterial pulse was well perceivable at a popliteal level and distally at the level of the dorsalis pedis and anterior tibial artery, with no sensory-motor impairment. We proposed placing the limb in traction and performing a total joint arthroplasty (TKA) subsequently. However, the patient refused the suggested treatment.

\section{Discussion}

Knee dislocation is a rare pathological condition that can be underestimated during the emergency assessment; therefore, X-rays, examination of vascular and neurological conditions, as well as a complete balance of articular injuries should be performed.

The immediate and stable closed reduction can lead to a remission of the neurovascular compression. However, dislocation, or subluxation of the knee, can be undiagnosed because of a diagnostic mistake at the time of the trauma, particularly in the absence of neurovascular injuries, which usually lead to surgical treatment.

Taylor et $\mathrm{al}^{4}$ reported good results with nonsurgical treatment. Conversely, Ríos et $\mathrm{al}^{1}$ recently demonstrated better functional results with surgical approach. Nonoperative treatment was associated with unsatisfactory results in $100 \%$ of the cases.

Chronic knee subluxation-dislocation is rare, as the severity of the injury always leads to an early diagnosis and to appropriate treatment during the acute phase. Sisto and Warren ${ }^{5}$ described a single case of chronic knee dislocation, treated 24 weeks after the acute injury with a reduction, and fixation with Steinmann crossed wires and pins. The 2-year follow-up showed a pain-free stable and rigid knee (range of motion: 5-40 degrees).

In the case described herein, the mechanism of knee dislocation mechanism was quite peculiar, as it was not caused by a violent trauma but by the acceleration of the substantial body mass during the fall. Therefore, there was no real joint dislocation at the time of trauma but a joint diastase with no immediate neurovascular injury. The detachment of the apex of the fibula's head attracted the attention of physicians that provided first aid, who neglected the more severe joint injury.
Also, unusual was the treatment both during emergency and follow-up; in fact, once the plaster was removed, the radiographic examination showed a worsening of the joint condition and no surgical solution was suggested.

Over the years, the load on the knee dramatically led to the dislocation becoming chronic and at the same time, it determined a slow adaptation of the popliteal neurovascular bundle, with an adaptive elongation that guaranteed adequate vascular supply to the leg, without any ruptures or occlusions.

The treatment of neglected knee dislocation may be performed by arthrodesis or arthroplasty. ${ }^{6}$ In this case, given the age of the patient (69 years), the compromised general health status and the modest functional needs, implanting a hinged TKA after a gradual reduction of the dislocation with the Ilizarov's apparatus appeared to be appropriate. Arthrodesis is not indicated in this case, whereas it is instead necessary in the presence of severe instability, paralysis, neuropathy, infections after TKA, and damage to the extensor mechanism. Although arthrodesis is a valid option, it can cause persistent knee pain, lower back pain, and difficulty in driving, with considerable limitations in resuming working activities. ${ }^{5}$

Unfortunately, the patient refused to undergo two surgeries, and therefore, this case is described as having no surgical solution.

\section{References}

1 Ríos A, Villa A, Fahandezh H, de José C, Vaquero J. Results after treatment of traumatic knee dislocations: a report of 26 cases. J Trauma 2003;55(03):489-494

2 Robertson A, Nutton RW, Keating JF. Dislocation of the knee. J Bone Joint Surg Br 2006;88(06):706-711

3 Henshaw RM, Shapiro MS, Oppenheim WL. Delayed reduction of traumatic knee dislocation. A case report and literature review. Clin Orthop Relat Res 1996;330(330):152-156

4 Taylor AR, Arden GP, Rainey HA. Traumatic dislocation of the knee. A report of forty-three cases with special reference to conservative treatment. J Bone Joint Surg Br 1972;54(01):96-102

5 Sisto DJ, Warren RF. Complete knee dislocation. A follow-up study of operative treatment. Clin Orthop Relat Res 1985;(198):94-101

6 Montgomery TJ, Savoie FH, White JL, Roberts TS, Hughes JL. Orthopedic management of knee dislocations. Comparison of surgical reconstruction and immobilization. Am J Knee Surg 1995;8(03):97-103 